\title{
Immediate breast reconstruction with expander following recurrent lesion resection and exchange to silicon breast implant in a pregnant triple negative breast cancer patient: case report
}

\author{
Yuko Kijima $^{1,2}$, Munetsugu Hirata ${ }^{1}$, Naomichi Higo ${ }^{1}$, Hiroko Toda ${ }^{1}$, Yoshiaki Shinden ${ }^{2}$, Zenichi Morise ${ }^{3}$, \\ Shoji Natsugoe ${ }^{2}$ \\ ${ }^{1}$ Department of Breast Surgery, Fujita Health University, School of Medicine, Toyoake, Aichi, Japan; ${ }^{2}$ Department of Digestive Surgery, Breast \\ and Thyroid Surgery, Kagoshima University Graduate School of Medical and Dental Sciences, Sakuragaoka, Kagoshima, Japan; ${ }^{3}$ Department of \\ Digestive Surgery, Fujita Health University, School of Medicine, Toyoake, Aichi, Japan \\ Correspondence to: Yuko Kijima, MD, PhD. Department of General Surgery, Breast Surgery, Fujita Health University, School of Medicine, 1-98 \\ Dengakugakubo, Kutsukakecho, Toyoake, Aichi 470-1192, Japan. Email: ykijima@fujita-hu.ac.jp.
}

\begin{abstract}
A 39-year-old gravida 1 para 1 pregnant Japanese woman underwent skin-sparing mastectomy and axillary lymph node dissection with immediate breast reconstruction (IBR) using a tissue expander (TE) at 32 weeks of pregnancy under general anesthesia. Inserted TE (300 cc) was expanded during breast feeding while the volume was $240 \mathrm{cc}$ of the resected breast tissue. One month after delivery, 2 months after surgery, the contralateral healthy breast increased in size and the inframammary line was deviated toward a caudal site which was larger than $300 \mathrm{cc}$-inflated TE. She stopped breast feeding to receive a systemic chemotherapy after one months-breast feeding. At 3 months after delivery, the healthy breast size was smaller than the 250 cc-expanded breast and both the inframammary lines were at the same level. She was diagnosed local recurrence 3 month-postoperatively, so we resected the recurrent lesion and exchanged TE to silicon breast implant immediately. Finally, a good symmetry was obtained after insertion of the 220 cc SBI. At an IBR using TE, we should know the dynamic change of breast volume and the level of inframammary line of the healthy breast during those phases of pregnancy, delivery, and nursing.
\end{abstract}

Keywords: Breast cancer; pregnancy; immediate reconstruction; silicon breast implant; case report

Submitted Feb 11, 2020. Accepted for publication Jan 24, 2021.

doi: $10.21037 / g s-20-217$

View this article at: http://dx.doi.org/10.21037/gs-20-217

\section{Introduction}

The rate of breast cancer during pregnancy (BCP) is expected to rise as more women delay childbearing (1). This observation is congruent with the known relationship between late parity, age, and breast cancer. Immediate breast reconstruction (IBR) after mastectomy for breast cancer is currently considered an essential component in managing breast cancer patients. However, only a few studies have evaluated the feasibility of IBR in patients of BCP (2-6).

It was difficult to know the change of breast size, shape, and the level of inframammary line during pregnancy. We here experienced a BCP patient received an IBR using TE. We learnt from her the dynamic change of breast volume and the level of inframammary line of the healthy breast during those phases of pregnancy, delivery, and nursing. This is the first report to describe in detail the operation procedure.

We present the following case in accordance with the CARE reporting checklist (available at http://dx.doi. org/10.21037/gs-20-217).

\section{Case presentation}

\section{Diagnosis}

A 39-year-old gravida 1 para 1 pregnant Japanese woman noticed a breast mass located on the outer-lower quadrant 
Table 1 The time line of pregnancy and breast cancer treatment

\begin{tabular}{lll}
\hline Date & Pregnancy & Diagnosis and treatment of breast cancer \\
\hline August, 20xx & 27 weeks of pregnancy & Notice a right breast mass \\
& 28 weeks of pregnancy & Diagnosis of breast cancer \\
September, 20xx & 31 weeks of pregnancy & Introduction to our hospital \\
& 32 weeks of pregnancy & SSM, Ax, immediate breast reconstruction using TE \\
October, 20xx & 37 weeks of pregnancy & Delivery \\
December, 20xx & & Diagnosis of local relapse, giving up breast feeding \\
January, 20xx+1 & Local resection of the recurrent lesion \\
February, 20xx+1 & Exchange TE to SBI \\
March, 20xx+1 & Postoperative systemic chemotherapy \\
December, 20xx+4 & Postoperative 4 years, Free from recurrence
\end{tabular}

SSM, skin sparing mastectomy; Ax, axillary lymph node dissection; TE, tissue expander; SBI, silicon breast implant.

area of the right breast at 27 weeks of pregnancy. She went to a hospital and was diagnosed as having invasive breast carcinoma by core needlle biopsy (CNB). She was referred to Kagoshima University for further examination of breast cancer during pregnancy (BCP) at 31 weeks of pregnancy. She had an experience of delivery and breast feeding at the age of 30 . She had no family history of breast cancer. Ultrasonography revealed that the mass lesion was located on the upper area of the right breast and there was no evidence of lymph node metastasis. CT and bone scintigraphy to detect distant metastases were not performed. She was diagnosed as having T1cN0Mx BCP, according to the TNM classification (7). The time line of this patient's procedure of pregnancy and breast cancer treatment were shown in Table 1.

\section{Immediate breast reconstruction (IBR) using tissue expander (TE)}

She underwent skin-sparing mastectomy and axillary lymph node dissection with IBR using a tissue expander (TE) at 32 weeks of pregnancy under general anesthesia (Figure 1A). The scarring caused by $\mathrm{CNB}$, which was located on the $3-\mathrm{cm}$ outer edge of the tumor, was removed at the same time. We placed the TE at a level $1 \mathrm{~cm}$ lower in comparison to the contralateral healthy breast (Figure 1B). The operation was performed without any postoperative complications for the patient or fetus. The volume of resected breast tissue was $240 \mathrm{cc}$. The pathological diagnosis of the breast cancer was invasive carcinoma, $18 \mathrm{~mm}$, and negative for estrogen receptor, progesterone receptor, and HER2 protein. No lymph node metastasis was seen in the resected 14 axillary lymph nodes. Finally, the patient was diagnosed as pT2N0M0 Stage IIA. There was no evidence of cancer implantation on the CNB scar or cancerous lesions on the resected breast tissue. We planned to add adjuvant systemic chemotherapy after a short period of breast feeding. The inserted expander volume was $300 \mathrm{cc}$, and $150 \mathrm{cc}$ of normal saline was inflated at the time of surgery.

\section{Diagnosis and treatment of local recurrence}

Four weeks after the breast surgery, a healthy baby was born by vaginal delivery at 37 weeks gestational age. One month after delivery, 2 months after surgery, the contralateral healthy breast increased in size (Figure 1C). The patient nursed for 2 months.

When the total expander volume inflation was $300 \mathrm{cc}, 15$ $\mathrm{mm}$ of mass was detected by palpation and ultrasonography at 3 months post-operation. It was located close to the resected CNB scar, and mobility was obtained in the subdermal area between the skin and the mass. Fine needle aspiration biopsy gave a positive result (Figure $2 A, B$ ). We suspected that it was local recurrence and performed local resection for the diagnosis and treatment. One centimeter of skin and parenchymal tissue from the edge of the mass were removed (Figure 2C). Fifty cc of normal saline was deflated. The results showed local recurrence of breast carcinoma and the deep part of the vertical edge was positive for cancer (Figure 2D,E,F). An ideal expansion was observed at 5 months post-operation and 

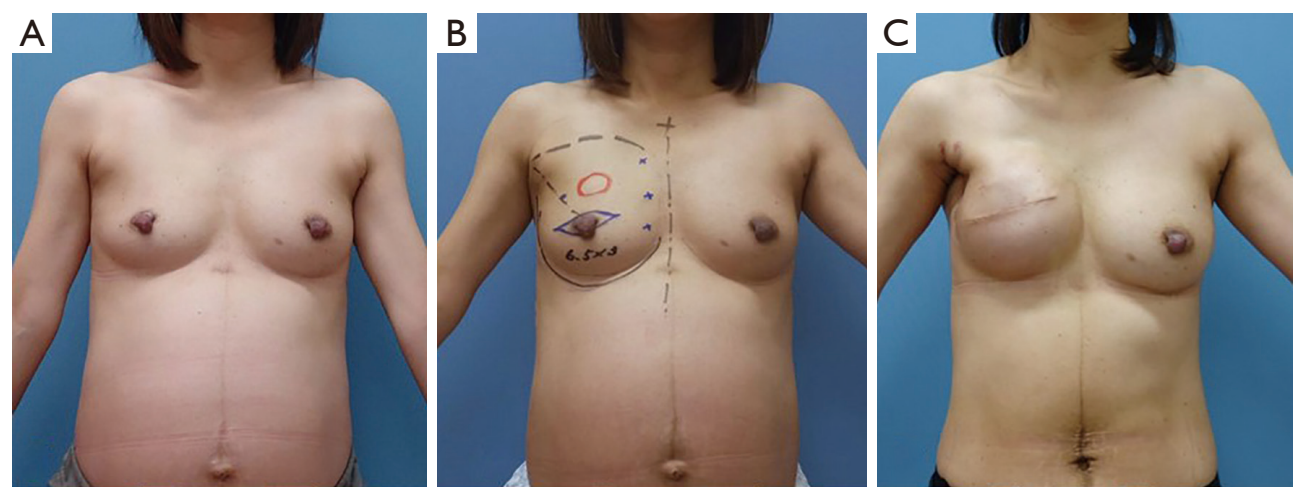

Figure 1 Gross findings pre- and post-delivery. (A,B) 37th week of pregnancy. (C) One month after delivery, 2 months after surgery. The contralateral healthy breast increased in size.
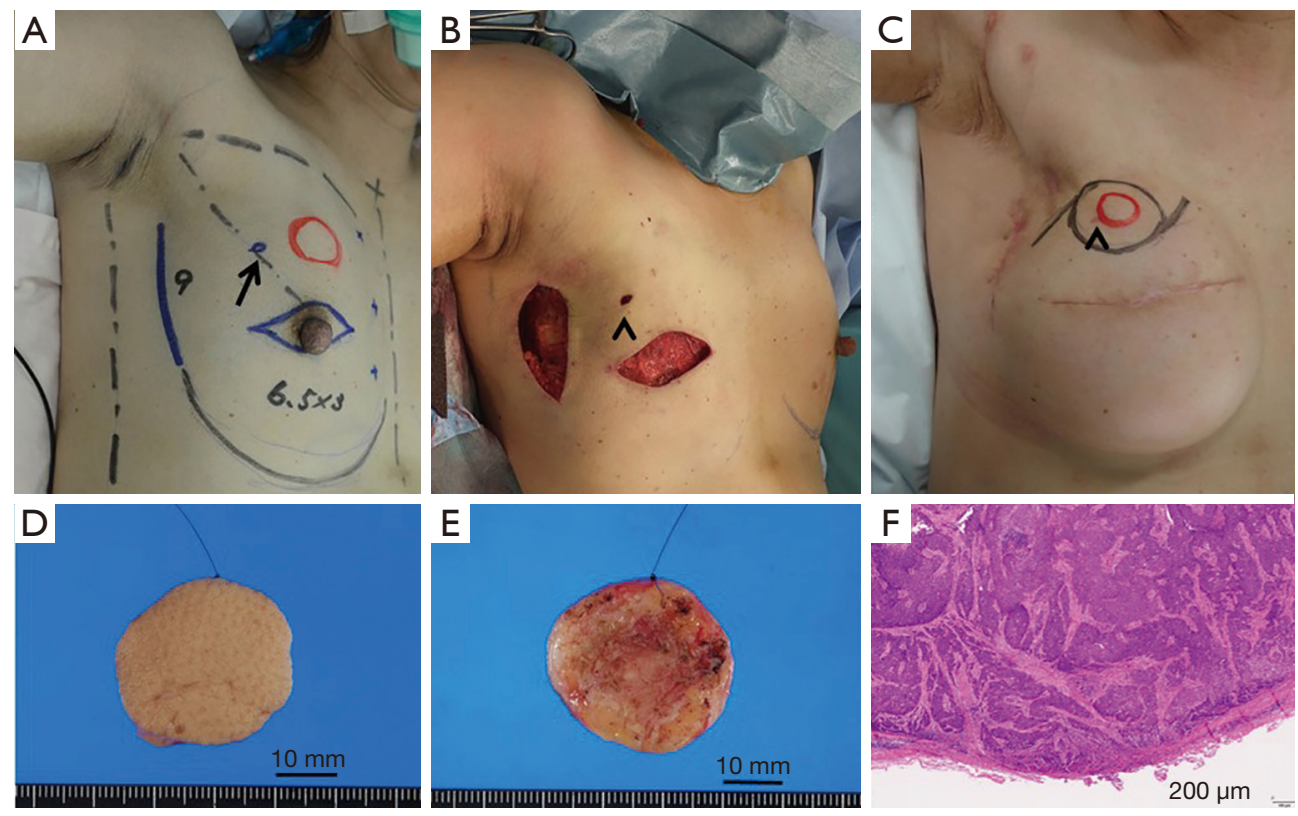

Figure 2 Gross findings before and after primary breast surgery and resected recurrent lesion. (A) Skin-sparing mastectomy and axillary lymph node dissection were performed with the double incision drawn in blue ink. Scarring due to CNB was removed (arrow). A cancerous lesion located on the upper area (red circle). (B) CNB scarring was removed completely (arrow head). (C) A subdermal local recurrence (red circle) was detected on the edge of the CNB scar (arrow head) 3 months after surgery. (D,E) One centimeter of skin and parenchymal tissue were obtained from the removed recurrent lesion. (F) Invasive breast cancer cells were detected with positive involvement of the vertical edge $(\mathrm{H} \& \mathrm{E}$ staining, original magnification $\times 100)$.

the patient's healthy left breast decreased in size (Figure $3 A, B$ ). A deformity due to local resection of the skin appeared on the upper-outer area of the expanded breast (Figure 3C).

\section{Exchange of ET for SIB}

We performed additional local resection of the right chest area, exchanged the TE for SBI, and reconstructed the nipple and areola. Prior to exchange of the TE for SBI, we performed local resection of the skin for repair of deformity and a part of major pectoral muscle was removed for additional resection of the cancerous lesion (Figure 4A). After removal of the TE and insertion of the SBI, the nipple and areola were reconstructed using inguinal-epidermis and half of the contralateral healthy 

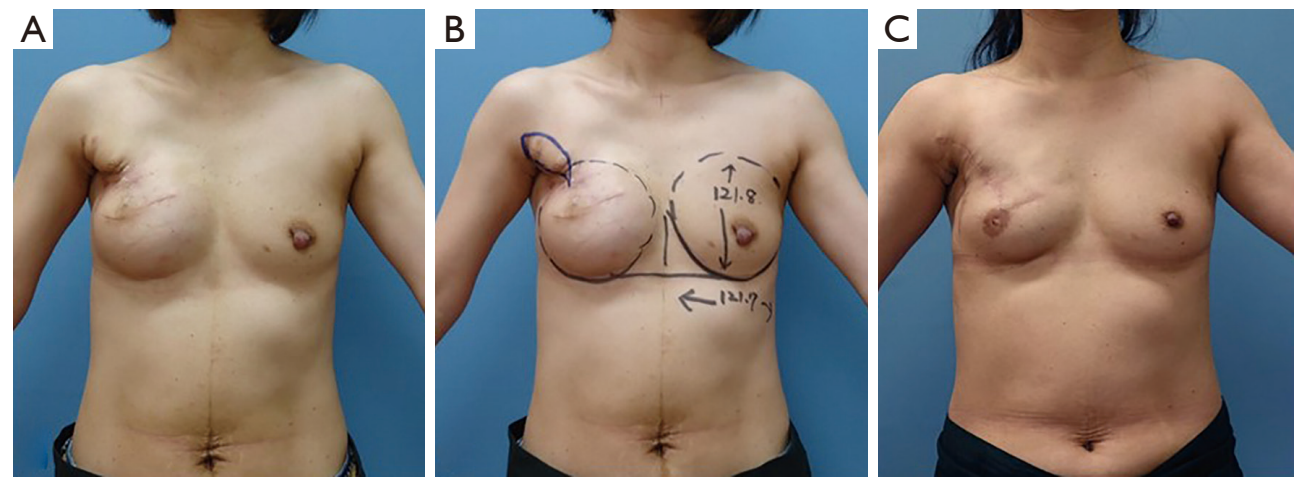

Figure 3 Gross findings post-delivery. (A,B) 1 month after resection of local recurrence, blue line, additional resection line of skin and subdermal tissue, (C) 3 years and 5 months after the initial breast surgery.
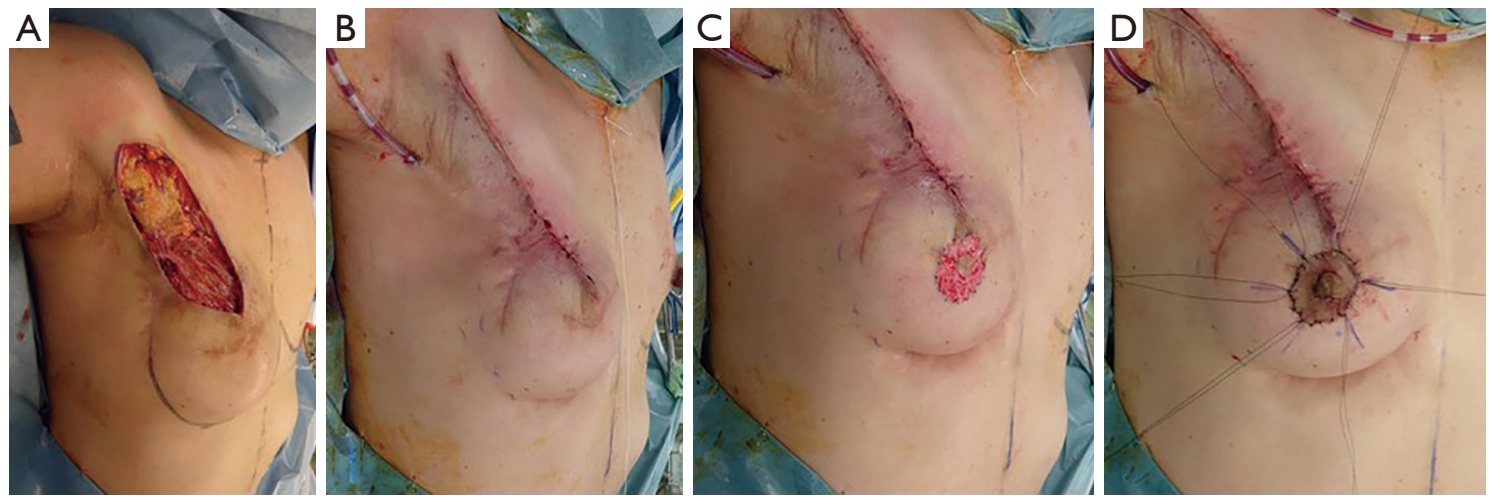

Figure 4 Additional resection at the time of removal of the TE and insertion of the SBI. (A) Prior to exchange of the TE for the SBI, skin and part of the major pectoral muscle were removed together. (B,C,D) After insertion of the SBI, the nipple and areola were reconstructed using inguinal-epidermis and half of the contralateral healthy nipple.

nipple (Figure 4B,C,D). Pathologically, there was no residual cancerous lesion on the additional resected tissue. The size of the inserted SBI was $220 \mathrm{cc}$. The patient underwent adjuvant systemic chemotherapy using epirubicin $\left(60 \mathrm{mg} / \mathrm{m}^{2}\right)$ and cyclophosphamide $\left(600 \mathrm{mg} / \mathrm{m}^{2}\right)$ once 3 weeks for 4 times, and docetaxel $\left(75 \mathrm{mg} / \mathrm{m}^{2}\right)$ once 3 weeks for 4 times after surgery. During and after chemotherapy, no systemic or local complications were seen. Four years after the initial breast surgery, the patient is free from recurrence and the cosmetic result is excellent.

\section{Ethical statement}

All procedures performed in studies involving human participants were in accordance with the ethical standards of the institutional and/or national research committee(s) and with the Helsinki Declaration (as revised in 2013). Written informed consent was obtained from the patient.

\section{Discussion}

Breast cancer during pregnancy (BCP) has been defined as cases of women diagnosed with breast cancer during pregnancy or within 1 year postpartum (8). It is currently the most common malignancy to occur during pregnancy, followed by cervical cancer, melanoma, and haematological malignancy (9). Some studies have found that BCP is more commonly diagnosed at an advanced stage because of increased breast density making clinical examinations and mammography more difficult to interpret (10-12).

Mastectomy was considered the standard surgical procedure in BCP for long time (13). Breast conservation can be selected for patients with BCP diagnosed during the third trimester. For them, breast conservation can be safely 
performed and postoperative radiotherapy can be postponed until after delivery without major concerns about a possible detrimental delay (14). On the other hand in patients with BCP diagnosed and operated in the first trimester, breast conservation performed during a very early gestational age is associated with a long delay in postoperative radiotherapy. Chen et al. reported a significant relationship between the waiting time for radiotherapy and local recurrence in a systemic review (15). While Amant et al. recommended that mastectomy should not be performed just because of the pregnancy itself, and breast conservation should be discussed whenever possible. In patients operated on during the third or even the second trimester, radiation therapy can be safely postponed until after delivery (16).

Currently, there are no available data concerning IBR for pregnant patients undergoing mastectomy for breast cancer. It is well known that IBR decreases the psychological impact of mutilation. That also provides a superior cosmetic outcome and better satisfaction compared to delayed reconstruction, respectively $(3,17,18)$. Although, the unpredictable physiologic changes of the breast during and after pregnancy makes it not suitable for IBR with definitive implant and contralateral reshaping. IBR with autologous tissue should not be considered due to the long operation time and increased risk of blood loss and postoperative complications.

During pregnancy, the contralateral breast size and position of the inframammary line and top of the breast mound dramatically changes. In the study by Lohsiriwat et al., seven patients had simultaneous contralateral breast procedures at the time of definitive implant substitution; three had additive mammoplasty and four had contralateral mastopexy (6). In the present case, the contralateral breast size was small without ptosis, so we felt it unnecessary to perform a contralateral operation to maintain symmetry to the reconstructed breast. However, it was difficult to determine the TE size and position, because the breast size, volume, and shape changes during pregnancy. As shown in Figure 1, the inframammary line of the healthy breast dramatically changed. At 1 month after delivery, the healthy breast was obviously larger than the 300 cc-expanded right breast (Figure 1C). At 3 months after delivery, the breast size was smaller than the 250 cc-expanded breast and both the inframammary lines were at the same level (Figure 1D,E). Finally, good symmetry was obtained after insertion of the 220 cc SBI (Figure 1F).

We add our present case to Lohsiriwat's case and summarized the clinical results as Table 2. Out of $13 \mathrm{BCP}$ patients, 9 completed expander inflation during pregnancy, and eventually underwent definitive implant positioning. The time from insertion to substitution of the expander was ranged from 10 to 32 months (6). Remaining four, our case was exchanged from TE to SBI after delivery and other three were still inflating their TE. They had a plan for definitive implant substitution procedure in the following months at the time of the literature submitted. We were able to observe the breast size and shape at pregnancy period, just after delivery, during breast feeding and after giving up breast feeding. From this experience we aware that it is adequate selection for a BCP patient with nonptotic and small breast to receive inflation of TE during pregnancy, after delivery and nursing. On the other hand for patients with ptotic and/or large breast, it would be necessary for them to add some reduction operations to the contralateral healthy breast to achieve a symmetrical result like Lohsiriwat's cases.

Two patients developed local recurrence and two other patients developed distant metastasis. Of the four patients diagnosed with local or distant recurrence, two were luminal type, one was HER2 type, and one was TN type (Table 2). The disease-free interval ranged from 3 to 39 months, which is relatively short. Especially in the present case, local recurrence due to needle tract implantation was detected only 3 months after surgery. In spite of there being no cancerous lesion on the resected CNB scar, recurrence occurred just under the dermis close to the CNB scar. In cases where the lesion is thought to have a high malignant potential, such as $\mathrm{TN}$, it may be necessary to remove the skin adjacent to a lesion surgically.

\section{Strength and limitation}

In this case, the breasts were not so large without ptosis even the late gestation. We aware that this procedure would not be bring excellent cosmetic outcome for a patient with large and/or ptotic breasts. For those patients, IBR using TE should be selected only the combination with a reduction mammoplasty of contralateral healthy breast.

Nevertheless, we experienced successful IBR during pregnancy, delivery, and nursing. The healthy breast size was dramatically changed during those periods. The volume of inserted TE was be able to be controlled due to add or gain the inserted normal saline, so we successfully selected an adequate size of SBI. Our report of the detailed operation procedure would be helpful for breast surgeons who might treat BCP-patient. 


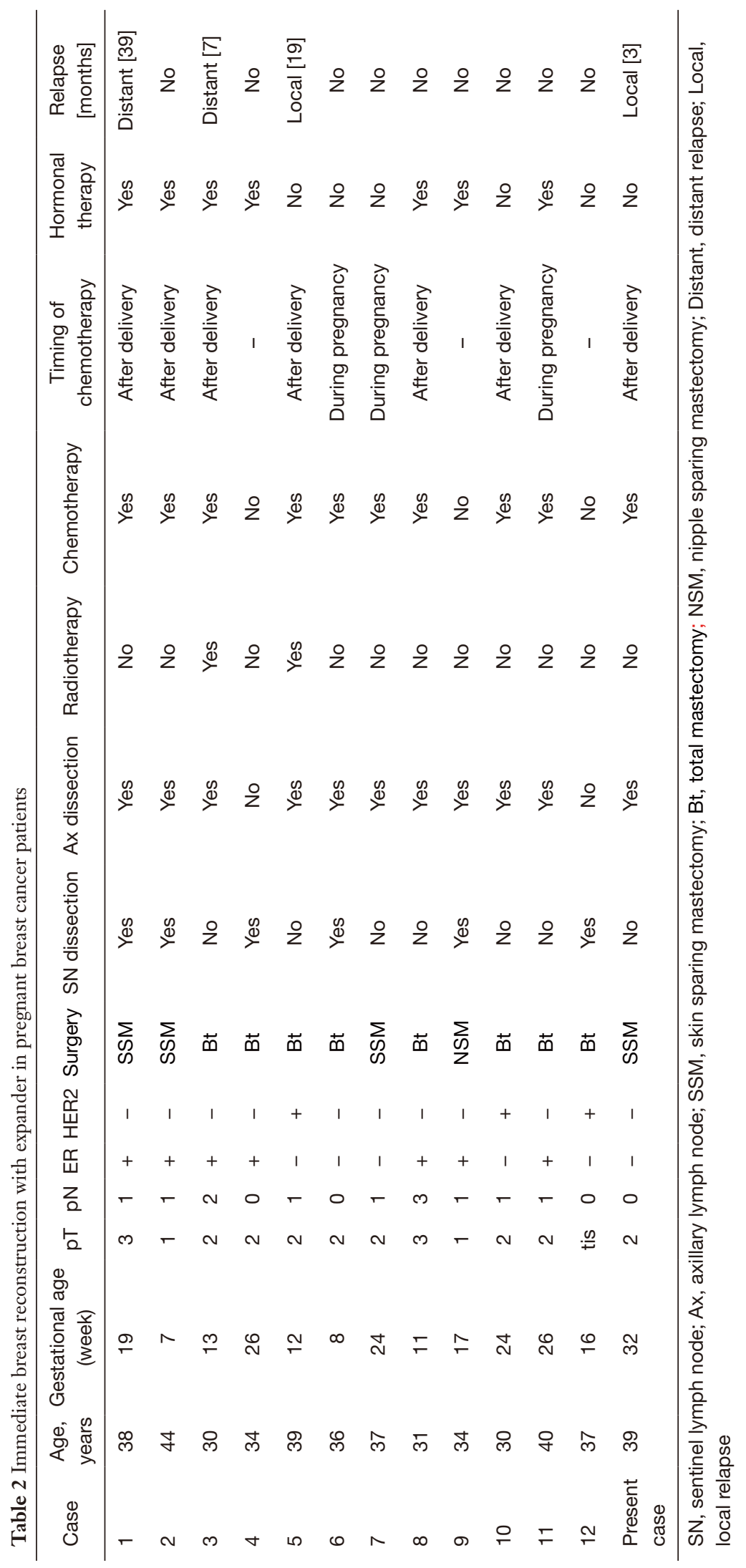




\section{Conclusions}

In this case of a patient diagnosed with BCP, local recurrence occurred after a short disease-free interval following IBR using TE. Oncologically, the needle tract feeding/implantation should be resected at the time of primary operation. The definitive observation of the breast during pregnancy, breast feeding, and after feeding would help for breast surgeons to reach a good symmetrical result.

\section{Acknowledgments}

Funding: None.

\section{Footnote}

Reporting Checklist: The authors have completed the CARE reporting checklist. Available at http://dx.doi.org/10.21037/ gs-20-217

Peer Review File: Available at http://dx.doi.org/10.21037/gs20-217

Conflicts of Interest: All authors have completed the ICMJE uniform disclosure form (available at http://dx.doi. org/10.21037/gs-20-217). Yuko Kijima serves as an unpaid editorial board member of Gland Surgery from Sep 2020 to Aug 2022. The authors have no conflicts of interest to declare.

Ethical Statement: The authors are accountable for all aspects of the work in ensuring that questions related to the accuracy or integrity of any part of the work are appropriately investigated and resolved. All procedures performed in studies involving human participants were in accordance with the ethical standards of the institutional and/or national research committee(s) and with the Helsinki Declaration (as revised in 2013). Written informed consent was obtained from the patient.

Open Access Statement: This is an Open Access article distributed in accordance with the Creative Commons Attribution-NonCommercial-NoDerivs 4.0 International License (CC BY-NC-ND 4.0), which permits the noncommercial replication and distribution of the article with the strict proviso that no changes or edits are made and the original work is properly cited (including links to both the formal publication through the relevant DOI and the license). See: https://creativecommons.org/licenses/by-nc-nd/4.0/.

\section{References}

1. Andersson TM, Johansson AL, et al. Increasing incidence of pregnancy-associated breast cancer in Sweden. Obstet Gynecol 2009;114:568-72.

2. Ho A, Cordeiro P, et al. Long-term outcomes in breast cancer patients undergoing immediate 2-stage expander/ implant reconstruction and postmastectomy radiation. Cancer 2012;118:2552-9.

3. Al-Ghazal SK, Sully L, et al. The psychological impact of immediate rather than delayed breast reconstruction. Eur J Surg Oncol 2000;26:17-9.

4. Petit JY, Gentilini O, et al. Oncological results of immediate breast reconstruction : Long term follow-up of a large series at a single institution. Breast Cancer Res Treat 2008;112:545-9.

5. Caragacianu DL, Mayer EL, et al. Immediate breast reconstruction following mastectomy in pregnant women with breast cancer. J Surg Oncol 2016;114:140-3.

6. Lohsiriwat V, Peccatori FA, et al. Immediate breast reconstruction with expander in pregnant breast cancer patients. Breast 2013;22:657-60.

7. Sobin LH, Gospoarowicz MK, et al. TNM classification of malignant tumours. 8th ed. New York: John Wiley \& Sons, Ltd. 2017.

8. Borges VF, Schedin PJ. Pregtnancy-assisted breast cancer: an entry needing refinement of the definition. Cancer 2012;118:3226-8.

9. Walton JR, Prasad PJ. Obestetric and neonatal outcomes of cancer treated during pregnancy. Clin Obstet Gynecol 2011;54:567-73.

10. Azim HA Jr, Santoro L, et al. Prognosis of pregnancyassociated breast cancer: a meta-analysis of 30 studies. Cancer Treat Rev 2012;38:834-42.

11. Ishida T, Yokoe T, Kasumi F, et al. Clinicopathologic characteristics and prognosis of breast cancer patients associated with pregnancy and lactation: analysis of casecontrol study in Japan. Jpn J cancer Res 1992;83:1143-9.

12. Anderson BO. Pregnancy-associated breast cancer. West J Med 1996;164:162.

13. Woo JC, Yu T, et al. Breast cancer in pregnancy: a literature review. Arch Surg 2003;138:91-8, discussion 99.

14. Duncan PG, Pope WDB, et al. Fetal risk of anesthesia and surgery during pregnancy. Anesthesiology 1986;64:790-4.

15. Chen Z, King W, et al. The relationship between waiting time for radiotherapy and outcome: a systemic review of the literature. Radiother Oncol 2008;87:3-16. 
16. Amant F, Deckers S, et al. Breast cancer in pregnancy: recommendations of an international consensus meeting. Eur J Cancer 2010;46:3158-68.

17. Morrow M, Jagsi R, et al. Surgeon recommendation and receipt of mastectomy for treatment of breast cancer.

Cite this article as: Kijima $Y$, Hirata $M$, Higo N, Toda H, Shinden Y, Morise Z, Natsugoe S. Immediate breast reconstruction with expander following recurrent lesion resection and exchange to silicon breast implant in a pregnant triple negative breast cancer patient: case report. Gland Surg 2021;10(5):1792-1799. doi: 10.21037/gs-20-217
JAMA 2009;302:1551-6.

18. Fernandez-Delgado J, Lopez-Pedraza MJ, et al. Satisfaction with and psychological impact of immediate and deferred breast reconstruction Ann Oncol 2008;19:1430-4. 